\title{
Influence of the Interorbital Interference on the Electron Tunneling in Scanning Tunneling Microscopy
}

\author{
L. JURCZYSZYN \\ Institute of Experimental Physics, University of Wrocław \\ pl. Maksa Borna 9, 50-204 Wrocław, Poland
}

\begin{abstract}
This article presents theoretical study of the influence of the interorbital interference on the electron tunneling in scanning tunneling microscopy. Detailed analysis shows that this kind of interference may modify significantly the tunneling current by the increase or decrease in the current contributions flowing through different orbitals of the surface atoms. This factor might cause the differences between the height and kind of scanning tunneling microscopy corrugation at different metal surfaces. This also might be a source of the unexpectedly high corrugation obtained from scanning tunneling microscopy measurements performed for some metal surfaces, which cannot be explained by the charge distribution along the substrate surface. The effects connected with the interorbital interference will be discussed in the context of the scanning tunneling microscopy simulations performed for $\mathrm{Ni}_{3} \mathrm{Al}(111)$ and (001) surfaces.

PACS numbers: 68.37.Ef, 73.20.-r, 73.40.Gk
\end{abstract}

\section{Introduction}

The final result of the measurements performed with the use of the scanning tunneling microscopy (STM) represents a very complex convolution of a large number of different factors. However, the starting point in the attempt of understanding of STM data is usually based on the assumption that the variation of tunneling current along sample surface directly reflects the local distribution of these electronic states from this surface which are involved in the tunneling process. This way of interpretation of experimental data is consistent with the idea of STM, and basically it works correctly, especially in the case of semiconductor surfaces, where the tunneling takes place through the electronic states well localized at the individual atoms of the surface structure. Therefore, the topographies 
of STM images of these surfaces are determined in most of the cases directly by the localization properties of the electronic states of the substrate that are active during the tunneling. Consequently, the theoretical description of the formation of these images can be performed in the framework of relatively simple methods, like for example the Tersoff-Hamann approach [1] — the STM images simulated in this way illustrate practically without significant modifications the distribution of the local density of states along the substrate surface. However, in some cases the understanding of the formation of STM images based on above arguments might be obviously insufficient for satisfactory explanation of STM data. It might take place especially in the case of metal surfaces: contrary to semiconductors, metal surfaces very often represent atomically smooth structures, and the tunneling takes place mostly through weakly localized electronic states. Consequently, STM images of metal surfaces usually have a very small corrugation and their topographies strongly depend on the number of different factors. In particular, the final result of STM measurements might be influenced by the interorbital interference connected with the electron tunneling through different orbitals of the atoms from the tip and the sample.

In this article we would like to discuss the role of such interference on the formation of STM image of the whole surface structure. The theoretical study presented in this paper is based on the STM simulations performed for $\mathrm{Al}(001)$ and $\mathrm{Ni}(001)$ surfaces with the use of different tips. The goal of this consideration is to clarify whether the interorbital interference can considerably modify the STM process.

\section{Model and method of calculations}

Theoretical study presented in this paper is based on the STM simulations performed in the framework of the non-equilibrium Green function formalism developed by Keldysh [2]. This formalism has been succesfully adopted for different theoretical investigations of electronic transport in microstructures [3, 4] and finally in the theory of STM [5]. It allows us to express the whole tunneling current as the coherent superposition on the current contributions flowing through different channels formed by different orbitals of the atoms from the tip and the sample.

Hamiltonian of the whole tip-sample system is represented by a sum of three terms that describe the tip $\left(\hat{H}_{\mathrm{T}}\right)$, sample $\left(\hat{H}_{\mathrm{S}}\right)$ and the interactions between them $\left(\hat{H}_{\mathrm{I}}\right)$ :

$$
\hat{H}=\hat{H}_{\mathrm{T}}+\hat{H}_{\mathrm{S}}+\hat{H}_{\mathrm{I}}
$$

The last term $\hat{H}_{\mathrm{I}}$ can be written as a sum of the hopping processes between the orbitals of the atoms forming the tip and the sample.

$$
\hat{H}=\sum_{\alpha j}\left[T_{\mathrm{TS}}(\alpha j) c_{\mathrm{T}}^{\dagger}(\alpha) c_{\mathrm{S}}(j)+T_{\mathrm{ST}}(j \alpha) c_{\mathrm{S}}^{\dagger}(j) c_{\mathrm{T}}(\alpha)\right] .
$$


Matrix $T_{\mathrm{TS}}$ that appears in above expression represents hoppings between different orbitals of the tip and the sample. When the considered system is in a stationary state (connected with the applied voltage), the tunneling current can be described by the following general formula [4]:

$$
J=(\mathrm{i} e / \hbar) \sum_{\alpha j}\left[T_{\mathrm{TS}}(\alpha j)\left\langle c_{\mathrm{T}}^{\dagger}(\alpha) c_{\mathrm{S}}(j)\right\rangle-T_{\mathrm{ST}}(j \alpha)\left\langle c_{\mathrm{S}}^{\dagger}(j) c_{\mathrm{T}}(\alpha)\right\rangle\right] .
$$

The applied Keldysh-Green function formalism lets us to pass from the expression (3) to the following equation for the tunneling current that allows us to perform STM simulations (more details are presented in [6]):

$$
\begin{aligned}
J= & (4 \pi e / \hbar) \int \operatorname{Tr}\left[\left(T_{\mathrm{TS}} \rho_{\mathrm{SS}}(\omega) D_{\mathrm{SS}}^{\mathrm{R}}(\omega) T_{\mathrm{ST}} \rho_{\mathrm{TT}}(\omega) D_{\mathrm{TT}}^{\mathrm{A}}(\omega)\right]\right. \\
& \times\left[f_{\mathrm{T}}(\omega)-f_{\mathrm{S}}(\omega)\right] \mathrm{d} \omega,
\end{aligned}
$$

where

and

$$
D_{\mathrm{SS}}^{\mathrm{R}}(\omega)=\left[I-T_{\mathrm{ST}} g_{\mathrm{TT}}^{\mathrm{R}}(\omega) T_{\mathrm{TS}} g_{\mathrm{SS}}^{\mathrm{R}}(\omega)\right]^{-1}
$$

$$
D_{\mathrm{TT}}^{\mathrm{A}}(\omega)=\left[I-T_{\mathrm{TS}} g_{\mathrm{SS}}^{\mathrm{A}}(\omega) T_{\mathrm{ST}} g_{\mathrm{TT}}^{\mathrm{A}}(\omega)\right]^{-1} .
$$

The above expressions (Eq. (4)) show that to calculate the tunneling current we need to find the matrices of the Green functions $\left(g_{\mathrm{TT}}^{\mathrm{A}}\right.$ and $g_{\mathrm{TT}}^{\mathrm{R}}$ for the tip, $g_{\mathrm{SS}}^{\mathrm{R}}$ and $g_{\mathrm{TT}}^{\mathrm{A}}$ for the sample) when both parts of the tip-sample system are uncoupled (i.e., for $T_{\mathrm{TS}}=0$ ). We also have to know the matrix $T_{\mathrm{TS}}$ of the hoppings between corresponding orbitals from the tip and the sample. In our study to calculate these hoppings we have used the expression for the Bardeen tunneling current between the atomic orbitals $\psi_{i}$ and $\psi_{j}$, multiplied by coefficient $\gamma[7]$ :

$$
T_{i, j}=-(\gamma / 2) \int \mathrm{d} S\left(\psi_{i} \nabla \psi_{j}-\psi_{j} \nabla \psi_{i}\right) .
$$

$\gamma$ in above expression typically takes values between 1.3 and 1.5 .

In the present consideration the description of STM tip has been performed with the help of the cluster-Bethe-lattice method [8]. In this approach the topmost part of the tip is represented by a pyramidal cluster of five atoms - one atom is located at the apex and four at the base of this pyramid. The influence of the rest of the tip is simulated by the Bethe lattice connected to each atom of this base. This allows us to calculate the Green function and density-of-states matrices of the tip (which are needed in Eq. (4)) by solving the reduced system formed by the atoms from the topmost pyramid. These calculations have been performed self-consistently by imposing the local charge neutrality condition at each atom of the apex cluster.

Electronic structure of the metal sample has been calculated in the framework of the self-consistent linear combination of atomic orbitals (LCAO) method described in detail in $[9,10]$. In this approach the Hamiltonian of the substrate is represented by the sum of two terms 


$$
H=H^{\mathrm{oe}}+H^{\mathrm{mb}}
$$

$H^{\circ e}$ represents the one-electron part

$$
H^{\mathrm{oe}}=\sum_{i, \delta} E_{i \delta} n_{i \delta}+\sum_{\delta,(i, j)} T_{i, j}^{\delta}\left(c_{i \delta}^{\dagger} c_{j, \delta}+c_{j, \delta}^{\dagger} c_{i, \delta}\right),
$$

while $H^{\mathrm{mb}}$ defines the many-body part of the whole LCAO Hamiltonian

$$
H^{\mathrm{mb}}=\sum_{i} U_{i} n_{i, \uparrow} n_{i, \downarrow}+\frac{1}{2} \sum_{i, j \neq i, \delta}\left(J_{i, j} n_{i, \delta} n_{j, \delta}+\hat{J}_{i, j} n_{i, \delta} n_{j, \delta}\right) .
$$

$E_{i \delta}$ represents here the different orbital levels, $T_{i, j}^{\delta}$ are the corresponding hopping interactions, while $U_{i}, J_{i, j}$, and $\hat{J}_{i, j}$ denote the intrasite and intersite Coulomb interactions between orbitals $i$ and $j$. As it was shown in [9], these Coulomb interactions can be calculated using the wave functions of the independent atoms that built up the considered system. In our study these Coulomb interactions have been obtained by using the wave functions of the independent atoms forming the system (for more details, see $[9,10]$ ). The many-body contributions are described within this LCAO formulation by using an extension of the local density approximation. It allows us to include such contributions constructing the Hartree and exchange-correlation potentials for each orbital defined by its occupancy and corresponding Coulomb interactions that appear in (8). This approach allows us to find self-consistently the occupancy of different orbitals and consequently, the distribution of the electronic charge in the whole considered system.

\section{Results and discussion}

The expression for the current tunneling in the tip-sample system (Eq. (4)) involves taking the trace of the multiplication of several matrices, which can be physically interpreted as a coherent superposition of electron tunneling through different channels formed by the orbitals of considered system. It means that this multichannel approach applied in our study allows us to consider different effects caused by the tunneling of electrons through different orbitals of the atoms from the tip and the sample.

In this paper we would like to focus our attention on the intra-atomic interference during the electron tunneling in tip-sample system. This kind of interference is directly connected with the corresponding intra-atomic off-diagonal elements of the Green functions and density-of-states matrices that appear in (4). It is known that for the atoms from the bulk of the crystal these intra-atomic off-diagonal elements are equal to zero. However, in the case of the atoms located in the crystal surface region this rule is not valid. It takes place for the orbitals that have significant component oriented perpendicular to the crystal surface. If we assume that $z$ axis represents direction perpendicular to this surface, the intra-atomic elements of type $s-p_{z}\left(p_{z}-s\right), s-d_{z}\left(d_{z}-s\right), p_{z}-d_{z}\left(d_{z}-p_{z}\right)$, 
and also $p_{x}-d_{x z}\left(d_{x z}-p_{x}\right), p_{y}-d_{y z}\left(d_{y z}-p_{y}\right)$ might be considerably different than zero. Therefore we may expect that the STM process could be influenced by the intra-atomic interference connected with the tunneling through these orbitals. On the other hand, the intra-atomic off-diagonal elements corresponding to orbital $s$ and these orbitals that are oriented parallel to the surface (like $p_{x y}$ for example) are always negligible even for surface atoms. The theoretical study presented in this paper is related only to the $s-p$ intra-atomic interference connected with the tunneling through these orbitals of the sample surface atoms.

The theoretical study presented in [11] clearly shows that the intra-atomic interorbitals interference connected with the tunneling through $s$ and $p_{z}$ orbitals of the surface atoms indeed influences significantly the tunneling of electrons in the tip-sample system. It has been shown that this factor might determine in large degree the topographies of obtained STM images. To demonstrate the role of such interference in STM process we will discuss in this paper the results of STM simulations performed for $\mathrm{Al}(001)$ and $\mathrm{Ni}(001)$ surfaces with the use of Al tip.

Figure 1 shows the STM simulations for Al(001) surface. It presents the variation of the conductance and its different contributions, when the Al tip is

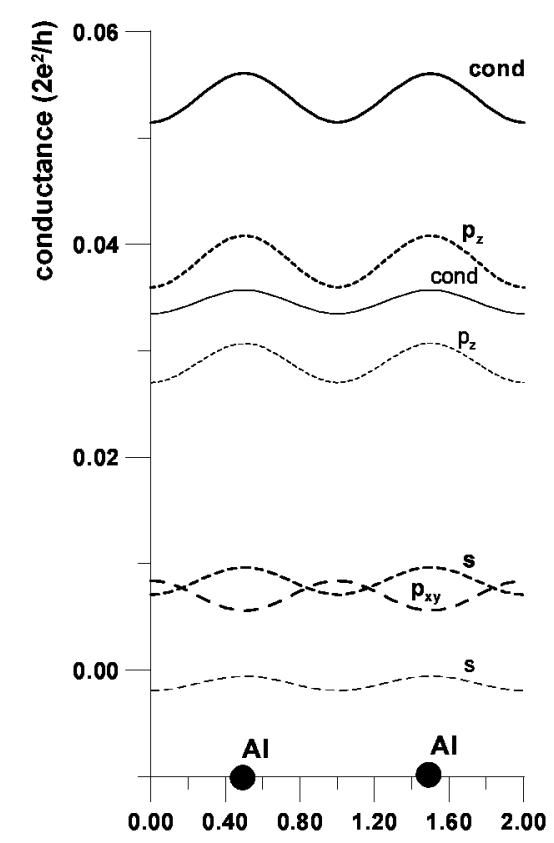

Fig. 1. Variation of the conductance and its contributions connected with the tunneling through $s, p_{z}$, and $p_{x y}$ orbitals of the surface atoms. Al tip moves along a dense-packed row of $\mathrm{Al}$ atoms from $\mathrm{Al}(001)$ surface. Tip sample distance equals to $4.8 \AA$. Tunneling takes place through the Fermi levels of $\mathrm{Al}$ tip and $\mathrm{Al}(001)$ surface. Thin lines represent the results obtained without intra-atomic $s-p_{z}$ interference. Values along horizontal axis are expressed in the units of the nearest-neighbour distance in the surface structure. 
moving along a dense-packed row of atoms in Al(001) surface. Tunneling takes place only between the Fermi levels of the sample and the tip. Simulations have been performed in constant-height mode with the tip-sample separation equal to $4.6 \AA$. Figure 1 presents the variation of current contributions connected with the tunneling through $s, p_{z}$, and $p_{x y}$ orbitals of the surface atoms. It follows from presented dependences that the variation of the total conductance presents normal corrugation (i.e. maxima appear above surface atoms), and this effect is caused by the current contributions connected with the tunneling through $s$ and $p_{z}$ orbitals of the surface $\mathrm{Al}$ atoms. It can be explained taking into account the localization properties of these orbitals $-p_{z}$ is oriented perpendicular to the surface, which causes that $p_{z}$ (and also $s$ ) contribution reach maximal values when tip is located directly above surface atom. On the other hand, the inverted corrugation of $p_{x y}$ contribution is caused by the parallel orientation of these orbitals with respect to the crystal surface.

To clarify the role of the intra-atomic $s-p_{z}$ interference, this simulation has been repeated removing selectively the off-diagonal elements from the Green functions and density-of-states matrices, that are connected with this kind of interference. The results obtained in this case are described in Fig. 1 by corresponding thin lines: these dependences clearly show that the removal of intra-atomic $s-p_{z}$ interference reduces considerably the $s$ and $p_{z}$ current contributions flowing through surface $\mathrm{Al}$ atoms. We can also notice that the relative variation of these contributions along the sample surfaces becomes visibly smaller. As a result, the average value and the variation of the total conductance, calculated without intra-atomic $s-p_{z}$ interference is much smaller than previously obtained (compare the corresponding thick and thin curves in Fig. 1). This means that in the considered case the $s-p_{z}$ interference increases the efficiency of the tunneling through these orbitals, and consequently increases the height of the STM corrugation along $\mathrm{Al}(001)$ surface.

However, theoretical study indicates that this interference may have also destructive influence on the STM process. This fact is well illustrated by the numerical results presented in Figs. 2 and 3. These two figures show the same kind of dependences as Fig. 1, but the tunneling takes place between electronic state from the Fermi level of Al tip and the states from the Al substrate that are located $7.5 \mathrm{eV}$ below and $7.8 \mathrm{eV}$ above its substrate Fermi level, respectively. These dependences clearly show that in both cases the intra-atomic $s-p_{z}$ interference has destructive influence on the tunneling through these orbitals. In the case shown in Fig. 2, the variation of the total conductance (thick solid line) presents now inverted corrugation (maxima appear between surface Al atoms). We can notice that this effect is caused by the current contribution connected with the tunneling through $p_{z}$ orbitals of the surface $\mathrm{Al}$ atoms (thick dotted curve): contrary to the case presented in Fig. 1, now the $p_{z}$ component has maxima located between surface Al atoms, which obviously cannot be explained by the localization prop- 


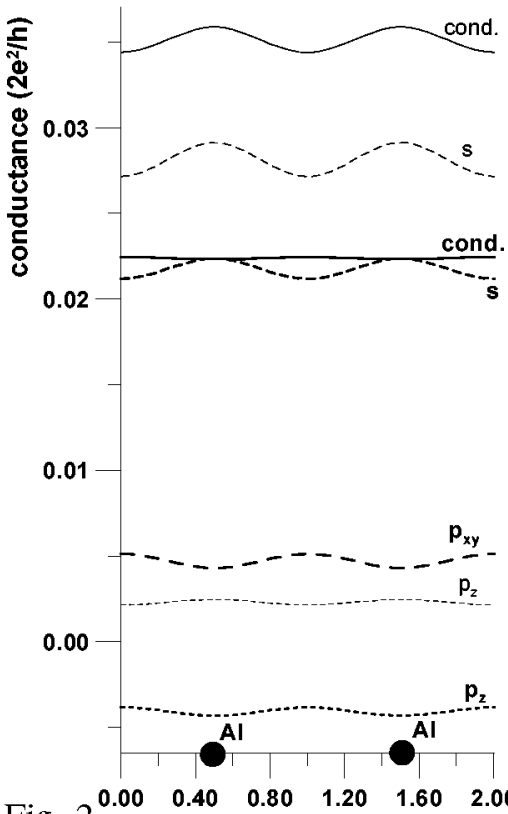

Fig. $2^{0.00}$

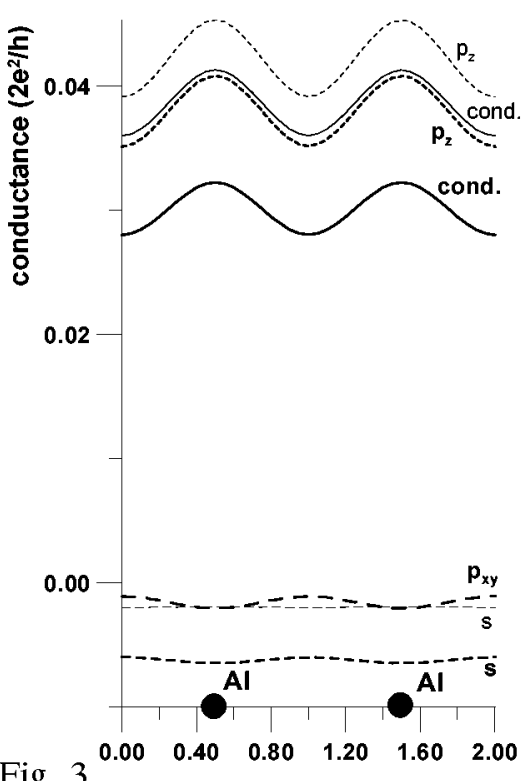

Fig. 3

Fig. 2. The same as in Fig. 1, but for the tunneling between the Fermi level of Al tip and the states of $\mathrm{Al}(001)$ located $7.5 \mathrm{eV}$ below the Fermi level of aluminum sample. Fig. 3. The same as in Fig. 1, but for the tunneling between the Fermi level of Al tip and the states of $\mathrm{Al}(001)$ located $7.8 \mathrm{eV}$ above the Fermi level of aluminum sample.

erties of $p_{z}$ orbitals. The removal of the intra-atomic $s-p_{z}$ interference increases significantly the efficiency of the tunneling through $s$ and $p_{z}$ orbitals of the surface atoms (compare the corresponding thin and thick lines in Fig. 2): instead inverted corrugation, $p_{z}$ contributions present now normal corrugation and correctly reproduce localization properties of these orbitals. Consequently, after removal of $s-p_{z}$ interference the total conductance increases its averaged value and changes its corrugation from inverted (solid thick line) to the normal one (solid thin line in Fig. 2).

The similar effect connected with intra-atomic $s-p_{z}$ interference appears in Fig. 3: the comparison of corresponding thin and thick lines in this figure demonstrates that the removal of this interference increases averaged values of $s$ and $p_{z}$ current components as well as their normal variations along the surface.

The obtained results show that intra-atomic $s-p_{z}$ interference may have the constructive or destructive influence on the STM process. The detailed analysis presented in [11] shows that this depends on the energies of the states from the sample that are active during the tunneling, with the respect to the potentials of $s$ and $p_{z}$ orbitals of the surface atoms denoted here by $E_{s}$ and $E_{p z}$, respectively (one-center integrals in LCAO language) — this has been schematically 


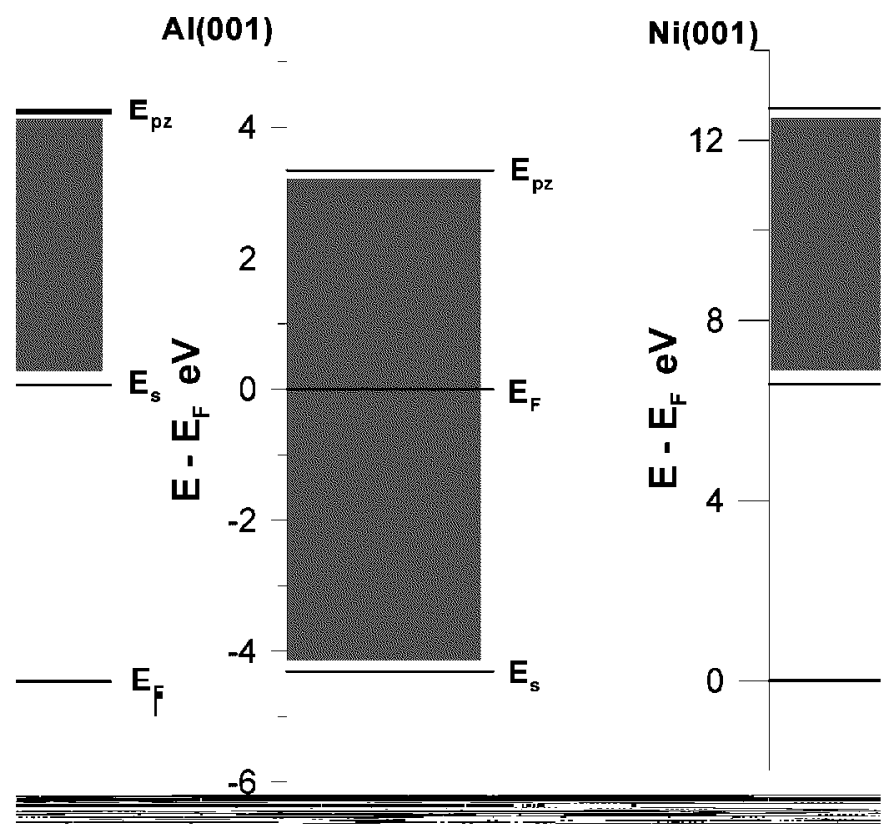

Fig. 4. The energy regions for $\mathrm{Al}(001)$ and $\mathrm{Ni}(001)$ surfaces, where the intra-atomic $s-p_{z}$ interference has constructive and destructive influence on the tunneling process. $E_{s}$ and $E_{p z}$ denote the potentials of $s$ and $p_{z}$ orbitals of the surface atoms, $E_{\mathrm{F}}$ is the position of the Fermi level.

illustrated in Fig. 4. When the tunneling in the tip-sample system takes place through the states from the sample with the energies located between $E_{s}$ and $E_{p z}$ levels (dark-gray region in Fig. 4), the intra-atomic $s-p_{z}$ interference increases the efficiency of the tunneling through $s$ and $p_{z}$ orbitals of the surface atoms: this interference increases the averaged value and the normal variations of $s$ and $p_{z}$ components along the surface. Consequently, this factor increases the value of the total conductance and causes a stronger normal corrugation of STM image. This situation corresponds with the case presented in Fig. 1, where the tunneling takes place through the state from the Fermi level of $\mathrm{Al}(001)$ surface. At this surface $E_{\mathrm{F}}$ is located $4.37 \mathrm{eV}$ above $E_{s}$ and $3.34 \mathrm{eV}$ below $E_{p z}$ levels of the surface $\mathrm{Al}$ atoms, so therefore in this case the intra-atomic $s-p_{z}$ interference has constructive influence on the STM process. On the other hand, for the tunneling through the states of the sample with energies lower than $E_{s}$ or higher than $E_{p z}$ (bright-gray regions in Fig. 4), this kind of interference destructively influences the electron tunneling in the tip-sample system. This situation is illustrated by Figs. 2 and 3, where the tunneling takes place through the states of $\mathrm{Al}$ substrate which are located well below $E_{s}$ or above $E_{p z}$ levels, respectively. In both cases the intra-atomic $s-p_{z}$ interference reduces significantly the value of $s$ and $p_{z}$ contributions flowing through 
surface $\mathrm{Al}$ atoms and their normal variation along the surface causing even its inverted corrugations. As a result, the inverted corrugation might appear in the whole STM image, as it is in Fig. 2.

It is also shown in Fig. 4 that in the case of $\mathrm{Ni(001)}$ surface the Fermi level is located almost seven electronvolts below $E_{s}$. Therefore, we may expect that contrary to $\mathrm{Al}(001)$ case, the tunneling between Fermi levels of $\mathrm{Ni}(001)$ surface and Al tip will be destructively influenced by intra-atomic interference connected with $s$ and $p_{z}$ orbitals of nickel surface atoms. This supposition is confirmed by the STM simulations performed for $\mathrm{Ni}(001)$ : the obtained results are presented in Fig. 5. These dependences clearly demonstrate that in this case the intra-atomic $s-p_{z}$ interference has destructive influence on the tunneling process reducing the value and variation of $s$ and $p_{z}$ components. As a consequence, because of this interference the value and the variation of the total conductance is considerably decreased (compare the corresponding thick and thin lines in Fig. 5).

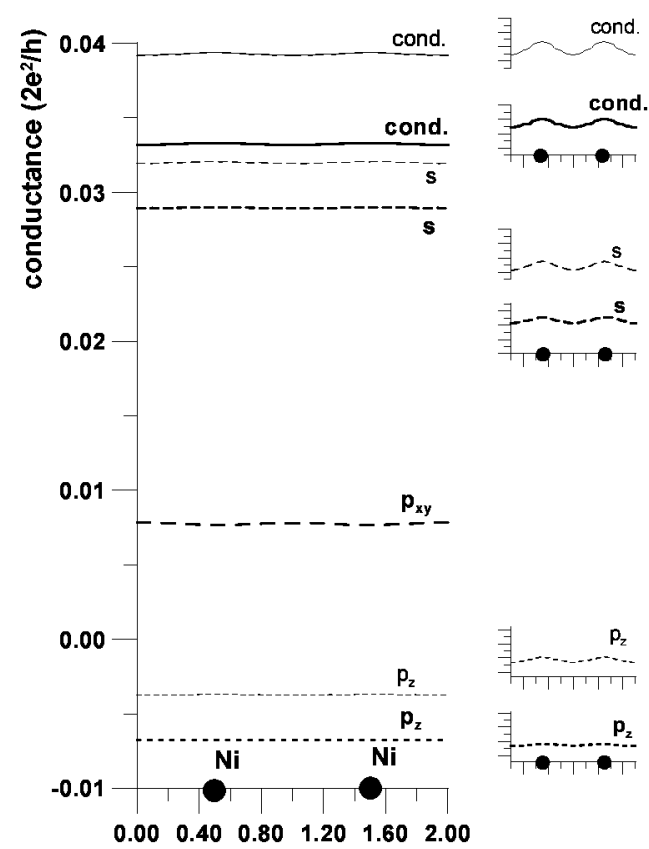

Fig. 5. The same as in Fig. 1 but for Ni(001) surface. Tip-sample distance is equal to $4.6 \AA$.

It follows from presented results that the influence of the intra-atomic $s-p_{z}$ interference on the tunneling through the Fermi levels of $\mathrm{Al}(001)$ and $\mathrm{Ni}(001)$ surfaces is opposite: in the first case (Fig. 1) it considerably increases the efficiency of the tunneling through $s$ and $p_{z}$ orbitals of the surface atoms, while for $\mathrm{Ni}(001)$ surface this interference reduces the tunneling through these orbitals. Theoretical 


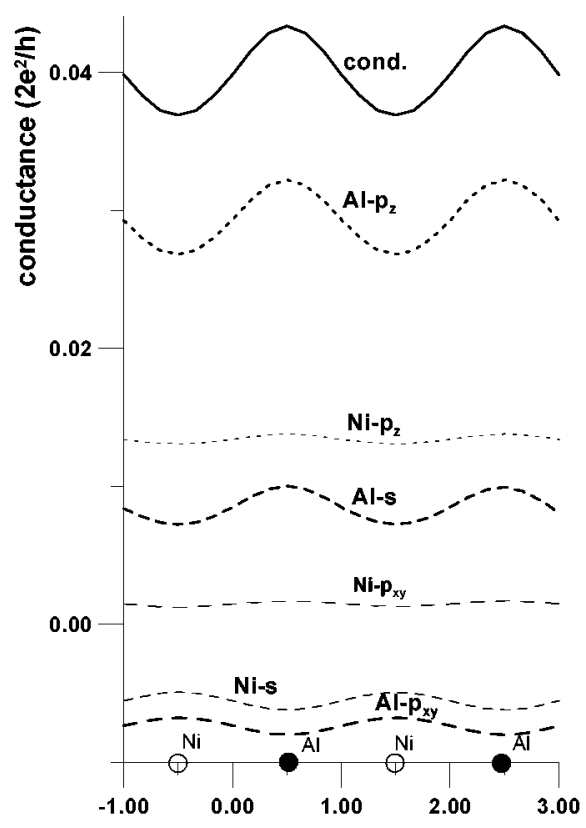

Fig. 6. Variation of the conductance for the Al tip moving along a dense-packed row of $\mathrm{Al}$ and $\mathrm{Ni}$ atoms of the $\mathrm{Ni}_{3} \mathrm{Al}(001)$ surface. Tip-sample separation equals to $5.0 \AA$. This figure presents the variation of the total conductance (solid line), as well as the $s, p_{z}$, and $p_{x y}$ contributions connected with the tunneling through the surface $\mathrm{Al}$ and $\mathrm{Ni}$ atoms.

study based on the STM simulations has shown that this factor may be responsible for the domination of $\mathrm{Al}$ atoms in STM images of $\mathrm{Ni}_{3} \mathrm{Al}$ surfaces [12, 13]. For the more stable configuration of $\mathrm{Ni}_{3} \mathrm{Al}(001)$ surface, the topmost atomic layer has a mixed structure with $50 \% \mathrm{Ni}-50 \% \mathrm{Al}$ composition, and STM simulations presented in this article have been performed for this structure. Figure 6 presents the variation of the conductance for the Al tip moving along a dense-packed row of $\mathrm{Al}$ and $\mathrm{Ni}$ atoms of $\mathrm{Ni}_{3} \mathrm{Al}(001)$ surface (tunneling between the Fermi levels of the tip and the sample). This figure also shows the variation of current contributions connected with the tunneling of electrons through $s$ and $p_{z}$ orbitals of the surface $\mathrm{Al}$ and $\mathrm{Ni}$ atoms (corresponding thick and thin curves, respectively). We can see that the total conductance has large maxima located above surface Al atoms, while the $\mathrm{Ni}$ atoms are invisible in this STM profile. Figure 6 shows that the changes of conductance are caused mainly by the tunneling through $s$ and $p_{z}$ orbitals of surface $\mathrm{Al}$ atoms. These current contributions have sharp maxima above Al atoms, which determines the shape of the whole STM profile. Figure 6 also presents the important differences between the current contributions connected with $s$ and $p_{z}$ orbitals of $\mathrm{Ni}$ and $\mathrm{Al}$ atoms. Figure 7 presents the density-of-states distributions of $s$ and $p_{z}$ states at the surface $\mathrm{Al}$ and $\mathrm{Ni}$ atoms of $\mathrm{Ni}_{3} \mathrm{Al}(001)$. 


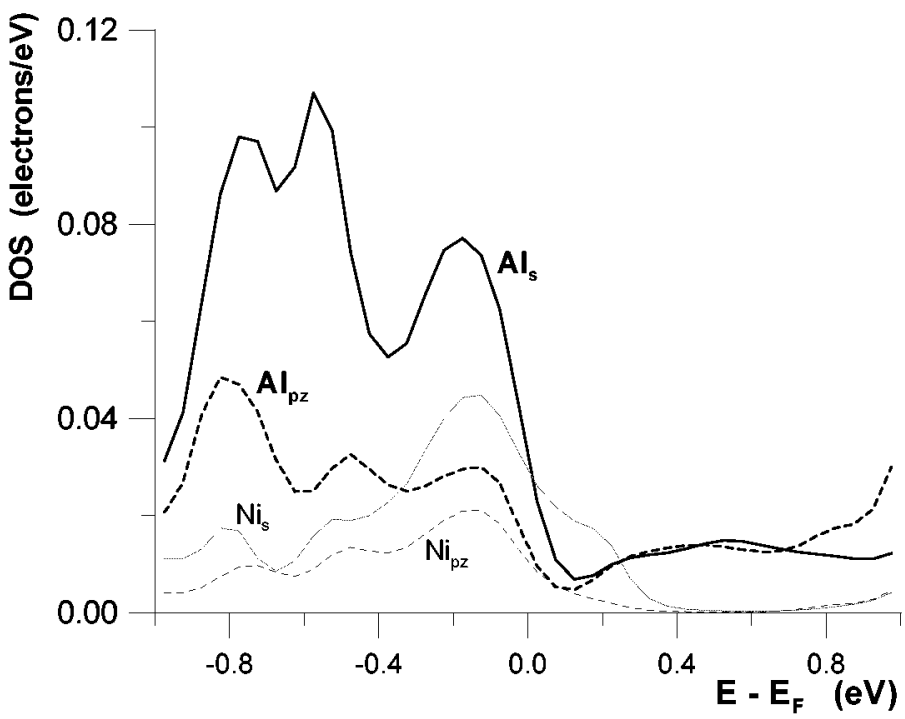

Fig. 7. Distributions of $s$ and $p_{z}$ states of surface $\mathrm{Al}$ and $\mathrm{Ni}$ atoms at $\mathrm{Ni}_{3} \mathrm{Al}(001)$ surface.

These distributions show that near the Fermi level the density of $p_{z}$ states at $\mathrm{Al}$ atoms is only $20 \%$ higher than it takes place for $\mathrm{Ni}$ atoms. However, as we can see in Fig. 6, the corresponding current contributions are very different: only $\mathrm{Al}-p_{z}$ component reproduces the localization properties of $p_{z}$ orbital showing the strong maxima above $\mathrm{Al}$ atoms ( $p_{z}$ orbitals are oriented perpendicular to the surface). The corresponding $\mathrm{Ni}-p_{z}$ contribution is almost flat with small maxima located above $\mathrm{Al}$ atoms, which of course cannot be explained via the localization properties of $\mathrm{Ni}-p_{z}$ orbitals. It follows from Fig. 7 that density of $s$ states at $\mathrm{Al}$ and $\mathrm{Ni}$ atoms is almost the same in the vicinity of the Fermi level. On the other hand, the corresponding $s$ current contributions connected with these atoms are drastically different. As we can see in Fig. 6, the Al-s component increases the current flowing through $\mathrm{Al}$ atoms. However, in the case of $\mathrm{Ni}$ atoms the $s$ contribution has a negative sign, which reduces considerably the current flowing through surface $\mathrm{Ni}$ atoms. As a result, the current flowing through $\mathrm{Ni}$ atoms is much more lower than it takes place for $\mathrm{Al}$ atoms: this effect cannot be explained taking into account only the density of states at particular surface atoms and the localization properties of the orbitals involved in the tunneling process.

Figure 8 presents the results of the STM simulation for the same case as in Fig. 6, but without intra-atomic $s-p_{z}$ interference related to the tunneling through $s$ and $p_{z}$ orbitals of the surface $\mathrm{Al}$ and $\mathrm{Ni}$ atoms. It follows from the comparison with Fig. 6 that dependences shown in Fig. 8 are basically different. As we can see, the removal of intra-atomic $s-p_{z}$ interference reduces considerably the $s$ and $p_{z}$ contributions flowing through surface $\mathrm{Al}$ atoms, and increases the corresponding contributions connected with $\mathrm{Ni}$ atoms: contrary to Fig. 6 , the Ni- $s$ and $\mathrm{Ni}-p_{z}$ 


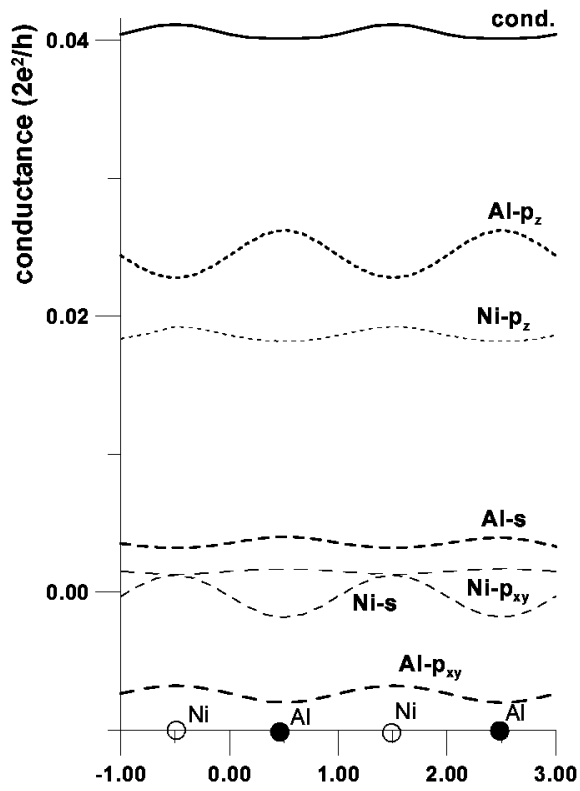

Fig. 8. The same as in Fig. 6, but without the intra-atomic $s-p_{z}$ interference.

components in Fig. 8 reproduce very well the localization of $\mathrm{Ni}$ atoms. As a result, the variation of the total conductance in Fig. 8 is basically different than in Fig. 6: now it is much smaller and the maxima appear above $\mathrm{Ni}$ atoms.

The effect connected with the removal of intra-atomic $s-p_{z}$ interference shown in Fig. 8 is entirely consistent with results presented earlier in Figs. 1 and 5 . The comparison of the results from Figs. 6 and 8 clearly shows that the domination of $\mathrm{Al}$ atoms in STM profile shown in Fig. 6 is directly caused by the intra-atomic $s-p_{z}$ interference connected with the tunneling through $s$ and $p_{z}$ orbitals of the surface atoms. This kind of interference reduces $s$ and $p_{z}$ current contributions flowing through Ni atoms, but on the other hand it increases considerably $s$ and $p_{z}$ contributions connected with surface $\mathrm{Al}$ atoms. Consequently, only $\mathrm{Al}$ surface atoms appear in STM profile (Fig. 6) and in the whole STM image, while Ni atoms are completely invisible. The theoretical study presented in [13] shows that the same mechanism leads also to the domination of Al atoms in STM images of $\mathrm{Ni}_{3} \mathrm{Al}(111)$ surface that was reported earlier in experimental work [12].

\section{Conclusions}

Theoretical study presented in this paper shows the important role of interorbital interference in the formation of STM images. The results of STM simulations clearly indicate that intra-atomic $s-p_{z}$ interference can significantly increase or decrease the efficiency of the tunneling through $s$ and $p_{z}$ orbitals of the surface atoms. It was shown that this effect depends on the energies of the electronic 
states of the sample that are active during the tunneling with respect to the potentials of $s$ and $p_{z}$ orbitals ( $E_{s}$ and $E_{p z}$, respectively). When the tunneling takes place through the states of the substrate which have energies higher than $E_{s}$ and lower than $E_{p z}$, the intra-atomic $s-p_{z}$ interference increases the efficiency of the tunneling through these orbitals of the surface atoms. This effect leads to the increase in the whole conductance and its normal variation along sample surface. On the other hand, the tunneling through the states of the sample with energies lower than $E_{s}$ or higher than $E_{p z}$ is connected with the destructive influence of intra-atomic $s-p_{z}$ interference on the tunneling through these orbitals. In these conditions, the value and the variation of $s$ and $p_{z}$ current contributions is reduced by such kind of interference: as it was shown, the destructive influence of $s-p_{z}$ interference might lead to the inversion of STM corrugation. It follows from presented results that this interference may cause the differences between the height and kind of STM corrugation at different metal surfaces. This factor might be a reason of the unexpectedly high corrugation provided by STM measurements of some metal surfaces, which cannot be explained by the charge distribution along these surfaces. We have also shown that the effects connected with interorbital interference can influence STM images of metal alloys by increasing and reducing the current contributions flowing through different kinds of surface atoms. This situation takes place in the case of $(001)$ and (111) surfaces of $\mathrm{Ni}_{3} \mathrm{Al}$ alloy: STM simulations performed for this case clearly demonstrate that intra-atomic $s-p_{z}$ interference is responsible for the domination of $\mathrm{Al}$ surface atoms in the STM images of these surfaces.

\section{References}

[1] J. Tersoff, D.R. Hamann, Phys. Rev. B 31, 806 (1985).

[2] L.V. Keldysh, Zh. Eksp. Teor. Phys. 47, 1515 (1964); Sov. Phys. JETP 20, 1018 (1965).

[3] C. Caroli, R. Combescot, P. Nozieres, D. Saint-James, J. Phys. C 4, 916 (1971).

[4] C. Caroli, R. Combescot, P. Nozieres, D. Saint-James, J. Phys. C 5, 21 (1972).

[5] J. Ferrer, A. Martin-Rodero, F. Flores, Phys. Rev. B 38, 10113 (1988).

[6] N. Mingo, L. Jurczyszyn, F.J. Garcia-Vidal, R. Saiz-Pardo, P.L. de Andres, F. Flores, S.Y. Wu, W. More, Phys. Rev. 54, 2225 (1996).

[7] E.C. Goldberg, A. Martin-Rodero, R. Monreal, F. Flores, Phys. Rev. B 39, 5684 (1989).

[8] L. Martin-Moreno, J.A. Verges, Phys. Rev. B 42, 7193 (1990).

[9] F.J. Garcia-Vidal, J. Merino, R. Perez, R. Rincon, J. Ortega, F. Flores, Phys. Rev. $B$ 50, 10537 (1994).

[10] F.J. Garcia-Vidal, A. Martin-Rodero, F. Flores, J. Ortega, R. Perez, Phys. Rev. $B$ 44, 11412 (1991).

[11] L. Jurczyszyn, Czech. J. Phys. 53, 19 (2003). 
[12] A. Rosenhahn, J. Schneider, C. Becker, K. Wandelt, Appl. Surf. Sci. 142, 169 (1999).

[13] L. Jurczyszyn, A. Rosenhahn, J. Schneider, C. Becker, K. Wandelt, Phys. Rev. B, to be published. 